\title{
Índice de cosecha con macro-nutrientes en grano de quinua (Chenopodium quinoa Willd)
}

\author{
Harvest index with macro-nutrients in quinoa grain (Chenopodium quinoa Willd) \\ Índice de colheita com macronutrientes em grão de quinoa (Chenopodium quinoa Willd) \\ - Marcelo Calvache Ulloa \\ mcalvache20@gmail.com \\ ORCID: 0000-0002-2438-5350 \\ Universidad Central del Ecuador, Ecuador \\ - Luciano Valle \\ correodelautor@investigación.com \\ ORCID: 0000-0001-8313-2302 \\ Universidad Técnica de Ambato, Ecuador
}

Artículo recibido 09 de octubre 2020 / Arbitrado y aceptado 30 de octubre 2020 / Publicado 04 de enero 2021

RESUMEN

En los Valles Interandinos del Ecuador entre 2000 y 3000 m.s.n.m se tienen bajos rendimientos de quinua con alto riesgo por la variabilidad del clima y la siembra de variedades criollas con inadecuadas prácticas de manejo. La investigación determinó las cantidades de nutrientes extraídos por tres variedades de quinua (Chenopodium quinoa Willd), durante el desarrollo del cultivo. El objetivo fue determinar las cantidades de nitrógeno, fósforo, potasio, calcio y magnesio que la planta de quinua extrae durante su ciclo de vida. Se evaluaron tres variedades de quinua, se determinó la cantidad de materia seca, porcentaje de nutrientes en estudio y cantidad de nutriente en $\mathrm{kg}$ ha- 1 de las muestras tomadas en hojas, tallos y panículas a los 20,40,60, 80, 100 y 120 días después de la siembra. Los resultados obtenidos indican que la variedad Imbaya con la dosis 150-80-40 de N, P, K mostro una mayor cantidad de materia seca acumulada en la planta total con $9870 \mathrm{~kg}$ ha-1 a los 120 días después de la siembra (dds). Los nutrientes más absorbidos por la planta de quinua fueron $\mathrm{K}$ y $\mathrm{N}$ seguido de $\mathrm{Ca}, \mathrm{Mg}$ y $\mathrm{P}$ en este orden. Los índices de cosecha (IC) obtenidos fueron: $\mathrm{N}=0,63$; $\mathrm{P}=0,64 ; \mathrm{K}=0,55 ; \mathrm{Ca}=0,52$ y $\mathrm{Mg}=0,65$. La aplicación del fertilizante en el cultivo de quinua se debe realizar en tres épocas: en la siembra, a la deshierba a los 30 días y al aporque a los 50 días de edad del cultivo.

Palabras clave: Acumulación; extracción; quinua; nutrientes
ABSTRACT

RESUMO
In the Inter-Andean Valleys of Ecuador between 2000 and 3000 meters above sea level, there are low quinoa yields with high risk due to the variability of the climate and the sowing of landraces with inadequate management practices. The investigation amounts of nutrients extracted by three varieties of quinoa (Chenopodium quinoa Willd) were determined during the development of the crop. The objetive was to determine the amounts of nitrogen, phosphorus, potassium, calcium and magnesium that the quinoa plant extracts during its life cycle. Three varieties of quinoa were evaluated, the amount of dry matter, percentage of nutrients under study and amount of nutrient in kg ha- 1 of the samples taken in leaves, stems and panicles were determined at $20,40,60,80,100$ and 120 days after sowing. The results obtained indicate that the Imbaya variety with the dose 150-80-40 of N, P, and K showed a greater amount of accumulated dry matter in the total plant with $9870 \mathrm{~kg}$ ha- 1 at 120 days after sowing (dds). The nutrients most absorbed by the quinoa plant were $\mathrm{K}$ and $\mathrm{N}$ followed by $\mathrm{Ca}, \mathrm{Mg}$ and $\mathrm{P}$ in this order. The harvest indices (IC) obtained were $\mathrm{N}=0.63$; $\mathrm{P}=0.64 ; \mathrm{K}=0.55 ; \mathrm{Ca}=0.52$ and $\mathrm{Mg}=0.65$. The application of the fertilizer in the quinoa crop must be carried out in three seasons: at sowing, at weeding at 30 days and at hilling at 50 days of crop age.

Key words: Accumulation; extraction; quinoa; nutrients
Nos Vales Interandinos do Equador, entre 2.000 e 3.000 metros acima do nível do mar, há baixos rendimentos de quinua com alto risco devido à variabilidade do clima e à semeadura de variedades locais com práticas de manejo inadequadas. $\mathrm{Na}$ presente investigação, as quantidades de nutrientes extraídas por três variedades de quinoa (Chenopodium quinoa Willd) foram determinadas durante o desenvolvimento da cultura. 0 objetivo foi determinar as quantidades de nitrogênio, fósforo, potássio, cálcio e magnésio que a planta da quinua extrai durante seu ciclo de vida. Foram avaliadas três variedades de quinoa, a quantidade de matéria seca, porcentagem de nutrientes em estudo e quantidade de nutriente em kg ha- 1 das amostras coletadas em folhas, caules e panículas foram determinadas a 20, 40, 60, 80, 100 e 120 dias após a semeadura. Os resultados obtidos indicam que a variedade Imbaya com a dose 150-80-40 de N, P, K apresentou maior quantidade de matéria seca acumulada na planta total com $9870 \mathrm{~kg}$ ha-1 aos 120 dias após a semeadura (dds). Os nutrientes mais absorvidos pela planta da quinua foram $\mathrm{K} \mathrm{e}$ $\mathrm{N}$ seguidos de $\mathrm{Ca}, \mathrm{Mg}$ e $\mathrm{P}$ nesta ordem. Os índices de colheita (IC) obtidos foram: $\mathrm{N}=$ 0,$63 ; \mathrm{P}=0,64 ; \mathrm{K}=0,55 ; \mathrm{Ca}=0,52$ e $\mathrm{Mg}=$ 0,65 . A aplicação do fertilizante na cultura da quinua deve ser realizada em três épocas: na semeadura, na sacha aos 30 dias e na amontoa aos 50 dias de idade de colheita.

Palavras-chave: Acumulação; extração; quinua; nutrientes 


\section{INTRODUCCIÓN}

En los Valles Interandinos del Ecuador entre 2000 y 3000 m.s.n.m se tienen bajos rendimientos del cultivo de quinua con alto riesgo por la variabilidad climática y la siembra de variedades criollas con inadecuadas prácticas de manejo como la fertilización de acuerdo a las necesidades del cultivo y los análisis químico de los suelos. La quinua (Chenopodium quinoa Willd), fue un alimento muy apreciado por las poblaciones aborígenes de Ecuador, por sus cualidades nutritivas y medicinales. Los Cañaris cultivaban esta planta antes de la llegada de los españoles (1).

En la actualidad a nivel mundial la quinua es valorada como el único alimento de origen vegetal que posee todos los aminoácidos esenciales, oligoelementos, vitaminas $\mathrm{y}$, no contiene gluten, es importante fuente de calcio, hierro y vitamina $B$, es decir la quinua es una buena fuente de muchos nutrientes (2-4). Los principales países productores de quinua son: Bolivia, Perú, Ecuador, Estados Unidos y Canadá, también se la cultiva en Inglaterra, Suecia, Dinamarca, los Países Bajos, Italia y Francia (5). Las zonas de producción de quinua en Ecuador se encuentran en las provincias de Carchi, Imbabura, Pichincha, Cotopaxi, Tungurahua y Chimborazo, el rendimiento promedio de la quinua alcanza 30 quintales por hectárea, según Peralta et al, (6), por debajo del rendimiento reportado por (7) que llega a 133 quintales por hectárea $(5985 \mathrm{Kg}$. ha), en la costa de Perú a una altitud de 1200 msnm.

El crecimiento de la planta de quinua está determinado por la genética de la planta y por factores bióticos y abióticos. Entre los abióticos, el factor nutrición de la planta se considera un aspecto determinante en el crecimiento y producción de la planta de quinua (8). La quinua es una planta exigente en nutrientes, principalmente de nitrógeno, calcio, fósforo y potasio, por ello requiere: un buen abonamiento y fertilización, dependiente de la riqueza y contenido de nutrientes de los suelos destinados a la siembra de quinua, de la rotación utilizada y también del nivel de producción que se desea obtener (9).

Para fertilizar el cultivo de quinua, Peralta, et al. (6), recomienda aplicar en suelos de baja fertilidad $80 \mathrm{~kg}$ de $\mathrm{N}$ y $40 \mathrm{~kg}$ de P2O5 por hectárea, aplicando el $20 \%$ del $\mathrm{N}$ a la siembra y el porcentaje restante a la deshierba o aporque, mientras que la totalidad del $\mathrm{P}$ aplicarlo en la siembra. Mientras que en suelos fértiles o luego de un cultivo de papa no recomienda usar fertilizante completo a la siembra, pero si aplicar $46 \mathrm{~kg}$ de $\mathrm{N}$ por hectárea al aporque.

Una curva de absorción de nutrientes es la representación gráfica de la cantidad de nutrientes extraídos por una planta durante su ciclo de vida, esta no constituye una herramienta de diagnóstico como el análisis foliar, más bien contribuyen a dar solidez a los programas de fertilización, debido a que constituyen las cantidades mínimas a las que el cultivo debe tener acceso para producir un determinado rendimiento. Conocer el comportamiento de estas curvas, permite identificar las épocas de mayor demanda y absorción nutrimental durante las diferentes etapas fenológicas de la planta (10-12). La extracción de nutrientes de los cultivos dentro de un mismo género no varía sustancialmente (13), pero cada curva es específica para cada variedad.

El Índice de Cosecha (I.C.) se obtiene dividiendo el peso de la semilla para el peso en seco de toda la planta y la FAO (7) encontró 
valores para quinua en promedio de 0,30 con variaciones de 0,21 a 0,45 dependiendo de las variedades. Sin embargo, no existe información disponible de los Índices de Cosecha con macro-nutrientes $\mathrm{N}, \mathrm{P}, \mathrm{K}, \mathrm{Ca}$ y $\mathrm{Mg}$ para granos de diferentes variedades de quinua $y$ diferentes fertilizaciones.

Mediante la presente investigación se proporcionará información técnica sobre la variación de absorción de $\mathrm{N}, \mathrm{P}, \mathrm{K}, \mathrm{Ca}$ y $\mathrm{Mg}$ en tres variedades de quinua, sometidas a tres fertilizaciones, durante su ciclo vegetativo, para establecer la dinámica de absorción de nutrientes y proyectar las épocas de aplicación adecuadas para potenciar los rendimientos y determinar los Índices de Cosecha con macronutrientes $\mathrm{N}, \mathrm{P}, \mathrm{K}, \mathrm{Ca}$ y $\mathrm{Mg}$ para este cultivo.

\section{MATERIALES Y MÉTODOS}

El experimento fue realizado en el Campo Docente Experimental La Tola (CADET) de la Universidad Central del Ecuador, Parroquia Tumbaco; Provincia de Pichincha, que se localiza a $78^{\circ} 23^{\prime} 30^{\prime \prime}$ longitud 0 y $00^{\circ} 13^{\prime} 30^{\prime \prime}$ latitud S, a $2465 \mathrm{msnm}$, con una temperatura promedio de $15,7^{\circ} \mathrm{C}$, temperatura mínima del aire $6,9^{\circ} \mathrm{C}$, temperatura máxima del aire 25,5 ${ }^{\circ} \mathrm{C}$, humedad relativa promedio de $74 \%$, brillo solar de $6.3 \mathrm{~h} /$ día y radiación solar equivalente a 4,3 $\mathrm{mm} /$ día, precipitación $890 \mathrm{~mm} \mathrm{y}$ humedad media anual del $75 \%$.

Según la clasificación americana, el suelo es un Typic Durandept (14), franco arenoso, con las características físicas y químicas de la Tabla 1.

Tabla 1. Resultados de análisis químico y físico de suelos al inicio del lote donde se llevó a cabo el experimento.

\begin{tabular}{llll}
\hline \multicolumn{1}{c}{ Parámetro } & Valor & Unidad & Interpretación \\
\hline Porosidad & 44.10 & $\%$ & \\
Densidad aparente & 1.48 & $\mathrm{~g} / \mathrm{cm} 3$ & Profundidad $30 \mathrm{~cm}$ \\
Contenido de agua & 16.99 & $\%$ & \\
$\mathrm{pH}$ & 7.40 & & Prac. Neutro \\
$\mathrm{CE}$ & 0.38 & $\mathrm{Mmhos} / \mathrm{cm}$ & Bajo \\
$\mathrm{M} .0$. & 1.44 & $\%$ & Bajo \\
$\mathrm{NH} 4$ & 24.10 & $\mathrm{ppm}$ & Bajo \\
$\mathrm{NO3}$ & 1.70 & $\mathrm{ppm}$ & Bajo \\
$\mathrm{P}$ & 51.80 & $\mathrm{ppm}$ & Exceso \\
$\mathrm{K}$ & 0.34 & $\mathrm{meq} / 100 \mathrm{ml}$ & Alto \\
$\mathrm{Ca}$ & 7.82 & $\mathrm{meq} / 100 \mathrm{ml}$ & Suficiente \\
$\mathrm{Mg}$ & 3.23 & $\mathrm{meq} / 100 \mathrm{ml}$ & Alto \\
$\mathrm{Na}$ & 0.09 & $\mathrm{meq} / 100 \mathrm{ml}$ & Bajo \\
$\mathrm{Cu}$ & 7.50 & $\mathrm{Ppm}$ & Exceso \\
$\mathrm{Fe}$ & 68.10 & $\mathrm{Ppm}$ & Alto \\
$\mathrm{Mn}$ & 3.80 & $\mathrm{Ppm}$ & Bajo \\
$\mathrm{Zn}$ & 4.90 & $\mathrm{Ppm}$ & Medio \\
$\mathrm{B}$ & 0.62 & $\mathrm{Ppm}$ & Bajo \\
$\mathrm{SO} 4$ & 10.20 & $\mathrm{Ppm}$ & Bajo \\
$\mathrm{Fe} / \mathrm{Mn}$ & 17.92 & $\mathrm{R} 1$ & Alto \\
$\mathrm{Ca} / \mathrm{Mg}$ & 2.42 & $\mathrm{R} 2$ & Alto \\
$\mathrm{Mg} / \mathrm{K}$ & 3.43 & $\mathrm{R} 3$ & Alto \\
$\mathrm{Ca}+\mathrm{Mg} / \mathrm{K}$ & 11.75 & $\mathrm{R} 4$ & Exceso \\
$\mathrm{CICE}$ & 12.08 & $\mathrm{meq} / 100 \mathrm{ml}$ & Medio \\
\hline
\end{tabular}


Los factores en estudio fueron: Tres niveles de fertilización química y tres variedades de quinua, en un arreglo factorial 3x3 que da 9 tratamientos (Tabla 2) con 4 repeticiones:

Tabla 2. Tratamientos evaluados en la investigación.

\begin{tabular}{ccl}
\hline Tratamientos & Nomenclatura & \multicolumn{1}{c}{ Descripción } \\
\hline T1 & v1f1 & Chasqui 00-00-00 kg N,P,K \\
T2 & v1f2 & Chasqui 75-40-20 kg N,P,K \\
T3 & v1f3 & Chasqui 150-80-40 kg N,P,K \\
T4 & v2f1 & Imbaya 00-00-00 kg N,P, K \\
T5 & v2f2 & Imbaya 75-40-20 kg N,P, K \\
T6 & v2f3 & Imbaya 150-80-40 kg N,P, K \\
T7 & v3f1 & Ecu-324 00-00-00 kg N,P,K \\
T8 & v3f2 & Ecu-324 75-40-20 kg N,P,K \\
T9 & v3f3 & Ecu-324 150-80-40 kg N,P,K \\
\hline
\end{tabular}

La siembra se realizó a chorro continuo con una distancia entre surcos de 0,60 m. Las parcelas experimentales fueron de $3 \mathrm{~m}$ de ancho por $10 \mathrm{~m}$ de largo, y en cada muestreo se tomaron 0,6 $\mathrm{m}$ de plantas, las mismas que fueron pesadas en húmedo, secadas en la estufa a $70{ }^{\circ} \mathrm{C}$, pesadas en seco y preparadas para los análisis químicos de los nutrientes.

Para cumplir con los objetivos trazados en la investigación se realizó la cuantificación de la producción de materia seca y la acumulación de $\mathrm{N}, \mathrm{P}, \mathrm{K}, \mathrm{Ca} \mathrm{y} \mathrm{Mg}$, en las muestras de hojas, tallos y panojas, recolectadas en las diferentes etapas fenológicas, basándose en la metodología de los laboratorios del INIAP SANTA CATALINA. Se realizaron los análisis y con los datos obtenidos se elaboraron curvas de absorción de los nutrientes en estudio y se determinaron los Índices de Cosecha (IC) del grano con $\mathrm{N}, \mathrm{P}, \mathrm{K}, \mathrm{Ca}, \mathrm{y} \mathrm{Mg}$. Se realizaron todas las labores culturales recomendadas por
Peralta y se aplicó riego por aspersión en 2 épocas de 30 días de sequia

En el estudio se evaluó tres variedades de quinua (Chasqui, Imbaya y ECU-324) y tres dosis de fertilización (0-0-0; 75-40-20; 15080-40 de N-P-K). Se utilizó un diseño de bloques completos al azar, en arreglo factorial 3 x 3 con cuatro repeticiones, para la comparación de medias se utilizó la prueba de tukey al 5\% de probabilidad.

Las variables en estudio que se midieron en todas las épocas de muestreo son las siguientes:

a) Producción de materia seca, en la cual se determinó el peso fresco de las muestras en el laboratorio, luego se colocaron estas muestras en la estufa para conocer su peso seco.

Para los cálculos correspondientes se utilizó la fórmula 1, 2,3 y 4 citadas por Calvache, et al (10). 
Fórmula 1. Cálculo del porcentaje de materia seca.

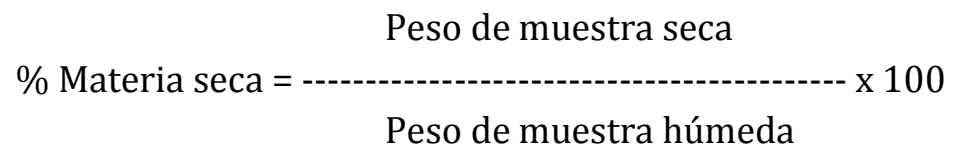

b) Biomasa acumulada:

Fórmula 2. Cálculo de la biomasa acumulada.

Ms $\left(\mathrm{kg} \mathrm{ha}^{-1}\right)=$ Materia seca x peso muestra húmeda $(\mathrm{kg} / \mathrm{ha})$

c) Acumulación de N, P, K, Ca y Mg.

La acumulación de los elementos en estudio se determinó en hojas, tallos y panículas. Sumando estos valores, se calculó la cantidad acumulada en toda la parte aérea de la planta, con ayuda de la siguiente ecuación:

Fórmula 3. Cálculo de la acumulación del nutriente.

Acumulación de Nutriente $\left(\mathrm{kg} \mathrm{ha}^{-1}\right)=$ Materia seca $(\mathrm{kg} / \mathrm{ha}) * \%$ Nutriente

Fórmula 4. Cálculo del índice de Cosecha (I.C.) para cada nutriente

En la cosecha, este parámetro se determinó dividiendo la cantidad de nutriente acumulado en el grano para la cantidad de nutriente acumulado en toda la planta.

\section{RESULTADOS Y DISCUSIÓN}

El resultado de la acumulación de nutrientes en el cultivo de quinua se interpreta de la siguiente manera:

\section{Producción de materia seca (Ms)}

El análisis de variancia de materia seca en la planta total (Tabla 3), mostró diferencias significativas para variedades y fertilización en todo el periodo del cultivo, para la interacción variedades por dosis de fertilización no se determinó significancia estadística a los 60 y 120 días después de la siembra. 
Tabla 3. Análisis de variancia para materia seca en planta total para tres variedades de quinua con tres dosis de fertilización en seis épocas de muestreo.

\begin{tabular}{|c|c|c|c|c|c|c|c|}
\hline \multirow{2}{*}{$\begin{array}{l}\text { Fuente de } \\
\text { variación }\end{array}$} & \multirow{2}{*}{$\begin{array}{c}\text { Grados } \\
\text { de } \\
\text { libertad }\end{array}$} & \multicolumn{6}{|c|}{ Cuadrados medios por muestreo en días después de la siembra (dds) } \\
\hline & & 20 & 40 & 60 & 80 & 100 & 120 \\
\hline Total & 35 & & & & & & \\
\hline Repeticiones & 3 & $61322,4^{* *}$ & $1382862,6^{\star *}$ & $743181,0^{\star *}$ & $11183500,1^{* *}$ & $40634465,0^{* \star}$ & $55644812,5^{\star \star}$ \\
\hline $\begin{array}{l}\text { Factor A } \\
\text { (Variedades) }\end{array}$ & 2 & $9959,9 * *$ & 376914,4 ** & $4900868,3^{* *}$ & 30309968,6 ** & $148443298,7^{* *}$ & $124911735,5^{\text {** }}$ \\
\hline $\begin{array}{l}\text { Factor B } \\
\text { (Fertilización) }\end{array}$ & 2 & 1804,0 ** & $450071,2^{* *}$ & 1544375,8 ** & 6854496,0 ** & $10927588,3^{* *}$ & $38949630,7^{* *}$ \\
\hline $\mathrm{AXB}$ & 4 & $472,1^{*}$ & 11441,2 * & $78996,6 \mathrm{~ns}$ & $1548293,5^{* *}$ & $2365267,3^{* *}$ & $1673045,0 \mathrm{~ns}$ \\
\hline $\begin{array}{l}\text { Error } \\
\text { experimental }\end{array}$ & 24 & 154,9 & 10150,8 & 78758,7 & 255825,4 & 1012727,8 & 1062467,2 \\
\hline $\begin{array}{l}\text { Coeficiente de } \\
\text { variación (\%) }\end{array}$ & & 6,53 & 11,14 & 13,39 & 14,47 & 15,32 & 13,22 \\
\hline
\end{tabular}

La fertilización que permitió una mayor acumulación de materia seca fue 150-80-40 kg ha-1 de N-P-K, obteniendo $11366,14 \mathrm{~kg} \mathrm{ha}^{-1}$ a los 120 dds, superando en 3533,27 y 6841,38 a los tratamientos $75-40-20$ y testigo absoluto (0-0-0), respectivamente (Tabla 4).
La variedad Imbaya mostró una mayor cantidad de materia seca acumulada en la planta total con $9870,16 \mathrm{~kg} \mathrm{ha}^{-1}$ a los $120 \mathrm{dds}$, superando en $3271,63 \mathrm{~kg} \mathrm{ha}^{-1}$ a la variedad Ecu-324 y en $2943,73 \mathrm{~kg} \mathrm{ha}^{-1}$ a la variedad Chasqui.

Tabla 4. Producción de materia seca $\left(\mathrm{kg} \mathrm{ha}^{-1}\right)$ en planta total de tres variedades de quinua con tres fertilizaciones, muestreadas en seis épocas.

\begin{tabular}{ccccccc}
\hline Factor & \multicolumn{5}{c}{ Fechas de muestreo en días después de la siembra (dds) } \\
\hline & $\mathbf{2 0}$ & $\mathbf{4 0}$ & $\mathbf{6 0}$ & $\mathbf{8 0}$ & $\mathbf{1 0 0}$ & $\mathbf{1 2 0}$ \\
Fertilización & & & & & & \\
$0-0-0$ & $166,60 \mathrm{c}$ & $712,70 \mathrm{c}$ & $1407,30 \mathrm{c}$ & $1835,00 \mathrm{c}$ & $3967,50 \mathrm{c}$ & $4524,76 \mathrm{c}$ \\
$75-40-20$ & $183,40 \mathrm{~b}$ & $948,90 \mathrm{~b}$ & $2226,80 \mathrm{~b}$ & $3650,80 \mathrm{~b}$ & $7065,40 \mathrm{~b}$ & $7832,87 \mathrm{~b}$ \\
$150-80-40$ & $221,70 \mathrm{a}$ & $1055,70 \mathrm{a}$ & $2659,70 \mathrm{a}$ & $5002,40 \mathrm{a}$ & $9943,70 \mathrm{a}$ & $11366,14 \mathrm{a}$ \\
Variedad & & & & & & \\
Chasqui & $187,50 \mathrm{~b}$ & $871,00 \mathrm{~b}$ & $1836,30 \mathrm{~b}$ & $3550,70 \mathrm{a}$ & $6816,60 \mathrm{~b}$ & $6926,43 \mathrm{~b}$ \\
Imbaya & $204,20 \mathrm{a}$ & $1114,20 \mathrm{a}$ & $2504,70 \mathrm{a}$ & $4223,10 \mathrm{~b}$ & $7654,20 \mathrm{a}$ & $9870,16 \mathrm{a}$ \\
Ecu-324 & $180,50 \mathrm{~b}$ & $732,00 \mathrm{c}$ & $1958,10 \mathrm{~b}$ & $2714,50 \mathrm{c}$ & $6505,60 \mathrm{~b}$ & $6598,53 \mathrm{~b}$ \\
\hline
\end{tabular}

Promedios con la misma letra dentro de cada factor y muestreo, son iguales estadísticamente, Tukey $(\mathrm{P}=0.05)$ 


\section{Acumulación de nitrógeno (N)}

La acumulación de nitrógeno presentó diferencias significativas en todo el periodo de muestreos y en el factor variedades. La planta de quinua muestra una baja acumulación de N (Figura 2) a los 20 dds, debido a la poca biomasa presente, a partir de los 20 dds hasta los 60 dds se evidencia un incremento de acumulación de $\mathrm{N}$, de los 60 a 80 dds continua la acumulación de $\mathrm{N}$, mientras que de los 80 a 100 dds se incrementa notablemente la acumulación de $\mathrm{N}$, al igual que de los 100 a $120 \mathrm{dds}$, continua la acumulación de N. Este comportamiento de incremento en la acumulación de $\mathrm{N}$ a partir de los 80 dds se sustenta en la mención que realiza CARE (8), El $\mathrm{N}$ en la planta se combina con componentes producidos por el metabolismo de carbohidratos para formar aminoácidos y proteínas. Siendo el constituyente esencial de las proteínas y está involucrado en todos los procesos principales de desarrollo de las plantas.

Solomon et al. (15), señala que las proteínas están formadas por aminoácidos y estos por un grupo amino $\left(-\mathrm{NH}_{2}\right)$ y un grupo carboxílico (-COOH), el $\mathrm{N}$ es constituyente del grupo amino, esto justifica la alta acumulación de $\mathrm{N}$ en la planta de quinua.

La variedad que más acumulo $\mathrm{N}$ (Tabla 5) fue la variedad Chasqui, con 201,73 kg N ha-1 a los 120 dds, seguida de la variedad Imbaya con $141,14 \mathrm{~kg} \mathrm{~N}$ ha-1 y finalmente se ubica la variedad Ecu-324 con 87,6 kg ha-1, a los 120 dds respectivamente. La fertilización con 15080-40 de N,P,K fue la que permitió una mayor acumulación de N con 204, $33 \mathrm{~kg}$. (Tabla 6).

Peralta, (1), en lo referente al $\mathrm{N}$ sugiere aplicar $80 \mathrm{~kg} \mathrm{ha}^{-1}$, lo que se aproximaría a la acumulación de $\mathrm{N}$ de la variedad 3 (Ecu-324).

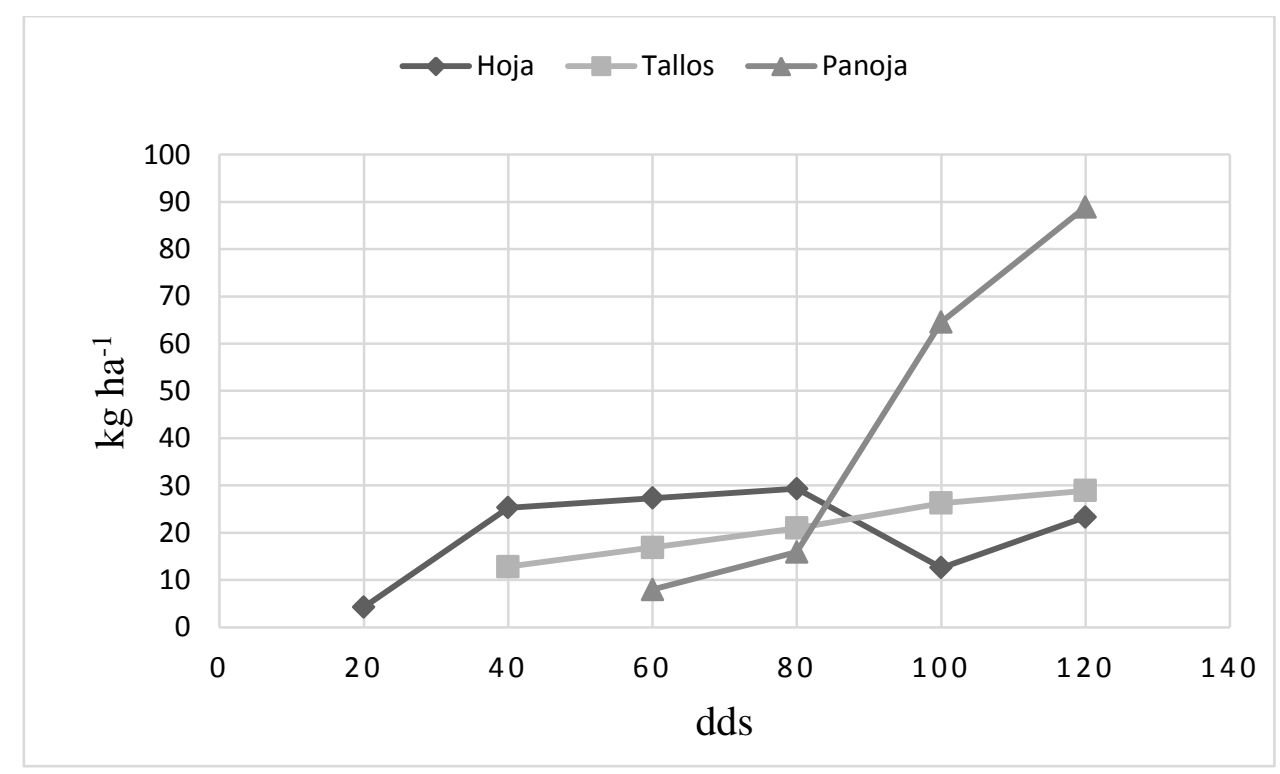

Figura 1. Curva de acumulación de nitrógeno en la planta de quinua (Chenopodium quinoa Willd), variedad Imbaya. 
El órgano que más acumulo $\mathrm{N}$ fue la panoja con $88,90 \mathrm{~kg} \mathrm{ha}^{-1}$, seguido del tallo con $28,93 \mathrm{~kg} \mathrm{ha}^{-1} \mathrm{y}$ finalmente las hojas con 23,32 $\mathrm{kg} \mathrm{ha}^{-1}$. La acumulación fue ascendente en la panoja y tallo, mientras que las hojas muestran un comportamiento ascendente de los 20 a 80 dds, para luego descender. A partir de los 80 días después de la siembra se evidencia como el $\mathrm{N}$ de las hojas se moviliza a la panoja donde albergan los granos. FAO (4), señala que la parte comestible de la quinua contiene del 10,4 al $17 \%$ de proteína, está proteína se compone por ocho aminoácidos los cuales están considerados esenciales tanto para niños como para adultos.

\section{Acumulación de fósforo (P)}

Del ADEVA se observó que para esta variable existen diferencias significativas en todas las fases de evaluación y en todos los factores e interacciones a los 80 y 120 dds. La acumulación de fósforo en la planta de quinua muestra un comportamiento ascendente durante todos los muestreos realizados, (Figura 2), de los 20 a 40 dds, la acumulación es baja; a los 60 y 80 dds se incrementa la acumulación y a los 100 y 120 dds, se incrementa vertiginosamente la acumulación de fósforo. CARE (8), señala que el P juega un papel importante en la transferencia de energía. Por eso es esencial para la fotosíntesis y para otros procesos químicofisiológicos. Es indispensable para la diferenciación celular y para el desarrollo de los tejidos, que forman los puntos de crecimiento de la planta. El fósforo es deficiente en la mayoría de los suelos del Ecuador. La variedad Ecu-324 acumula mayor cantidad de $\mathrm{P}$, con un valor de 41,29 kg P ha1 , seguida de la variedad Imbaya con $37,8 \mathrm{~kg}$ $\mathrm{P}$ ha-1 y la variedad Chasqui con $37,55 \mathrm{~kg} P$ ha-1 respectivamente. (Tabla 5). Peralta et al (6), recomiendan aplicar $40 \mathrm{~kg}$ ha-1 de $\mathrm{P}$, lo que se ajustaría al requerimiento de las tres variedades en estudio.

La panoja fue el órgano que más acumulo P con 24,41 kg ha-1. FAO (4), menciona que los granos de quinua contienen un promedio de $383,7 \mathrm{mg}$ por cada 100 gramos de peso seco. El tallo acumuló 9,11 $\mathrm{kg}$ ha-1 y finalmente se ubica las hojas con 4,28 kg ha-1. En las hojas se evidencia una acumulación sostenida de los 20 a 80 dds, posterior a esta edad ocurre una leve disminución. En el tallo se produjo una importante acumulación de los 40 a 80 dds, mientras que a los 100 y 120 dds, la acumulación evidencio un ligero incremento. Y en la panoja la acumulación es baja de los 60 a $80 \mathrm{dds}$, por cuanto es el inicio de esta etapa fenológica, mientras que a los 100 y 120 dds se produce un fuerte incremento de acumulación de P. A partir de los 80 dds se observa que el $\mathrm{P}$ de las hojas se moviliza a la panoja, mientras que el tallo a partir de esta edad detiene el ritmo de acumulación que venía realizando. Solomon, et al. (15), señala que el $P$ es parte esencial de los procesos que transfieren el código genético de una generación a otra, por lo que se observa que la acumulación de $P$ se incrementa a partir de los 80 dds que corresponde a la floración. También el P está. 


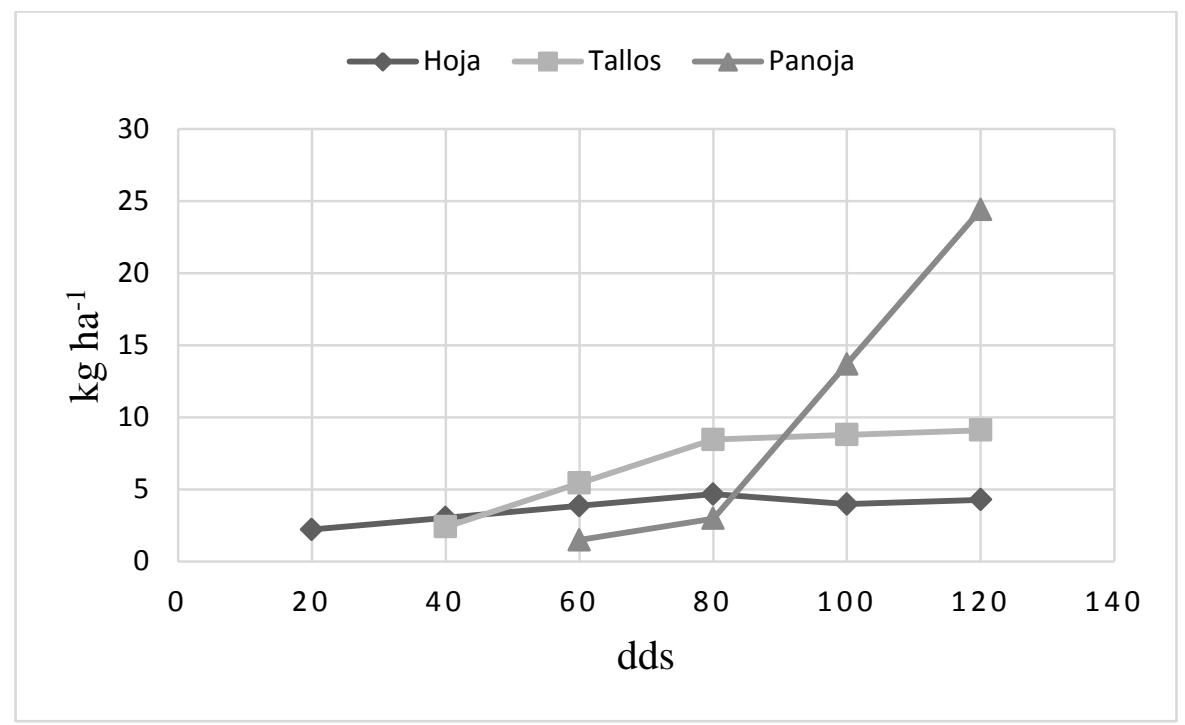

Figura 2. Acumulación de P en planta de quinua (Chenopodium quinoa Willd), variedad Imbaya

\section{Acumulación de potasio (K)}

Del análisis de varianza de acumulación de potasio se observó diferencias significativas en todo el periodo de evaluación. En la planta de quinua, (Figura 3) de los 20 a 40 dds se observa baja acumulación; en la segunda etapa de los 40 a 100 y 120 dds, se incrementa la acumulación de potasio. Este importante incremento de $\mathrm{K}$ en la planta se debe a que este elemento químico activa más de 60 enzimas; por ello juega un papel vital en la síntesis de carbohidratos y de proteínas (8).

Como se observa en la Tabla 5, la variedad Ecu-324, acumula mayor cantidad de K, con un valor de $341.5 \mathrm{~kg} \mathrm{~K}$ ha-1, seguida de la variedad Imbaya con 220,78 kg K ha-1 a los 120 dds y la variedad Chasqui con 106,60 kg K ha-1 a los 100 dds. FAO et al (7) presenta un valor de $585 \mathrm{~kg}$ ha-1 de absorción de $\mathrm{K}$ en la planta total de quinua, lo que corrobora los valores obtenidos en esta investigación. En la Tabla 6 se demuestra que la fertilización potásica si tuvo un efecto significativo, pues la dosis de 150-80-40 de N, P, K produjo la mayor acumulación de $\mathrm{K}$ en la planta con 337,39 Kg., mientras que la testigo solamente acumulo $124,37 \mathrm{~kg}$.
Villavicencio et al (16), recomienda no aplicar $\mathrm{K}$ en la fertilización del cultivo de quinua, lo que no corresponde a la cantidad importante que absorbe de este nutriente la planta de quinua.

La acumulación de potasio (Figura 3) fue mayor en la panoja con 274,32 kg ha-1, donde se encuentran los granos. Según FAO (4), la quinua es un alimento que aporta una importante cantidad de potasio, con un contenido de $926,7 \mathrm{mg}$ por cada $100 \mathrm{~g}$ de peso en seco. En menor cantidad se acumula en el tallo con 70,51 kg ha-1 y en las hojas con 71,27 $\mathrm{kg}$ ha-1. El K es vital para la fotosíntesis por lo que se observa un incremento paulatino en las hojas debido al aumento de la biomasa, mientras que en el tallo la acumulación es alta hasta los 80 dds, posterior a esa edad la acumulación en el tallo disminuye. Mientras que en la panoja desde los 80 dds el K aumenta su acumulación debido a su participación en la síntesis de proteínas que van a almacenarse en los granos de quinua, que contienen del 10,4 al $17 \%$ de proteína (4). 


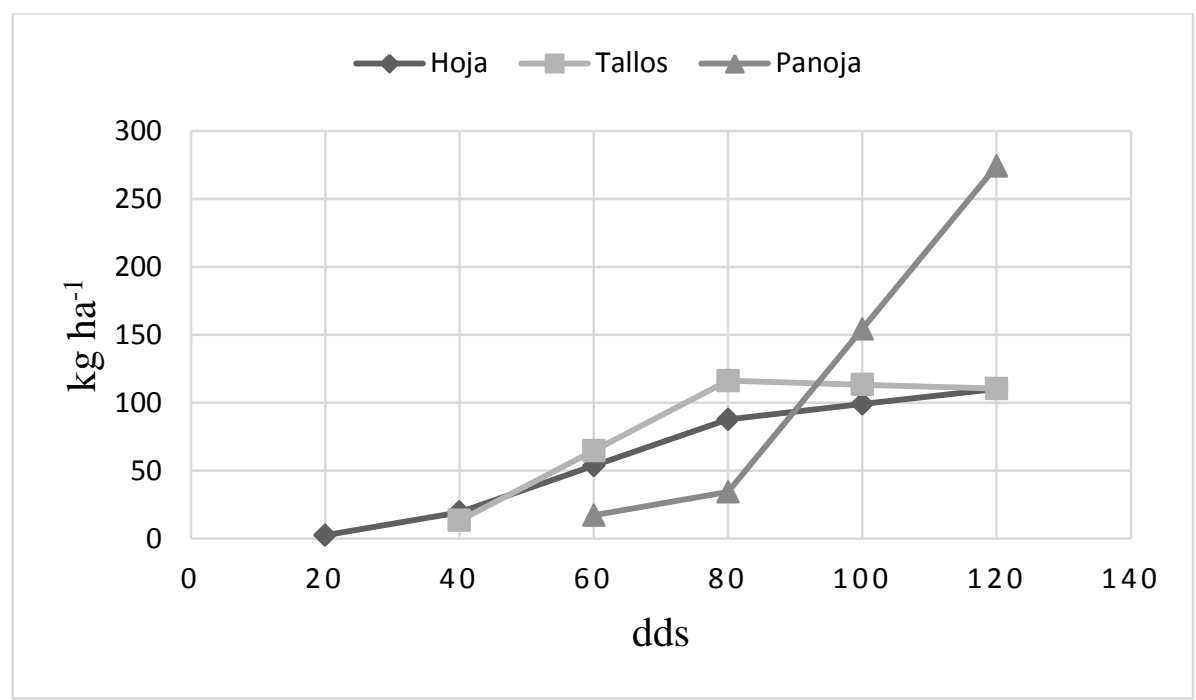

Figura 3. Acumulación de K en planta de quinua (Chenopodium quinoa Willd), variedad Imbaya.

\section{Acumulación de calcio (Ca)}

El ADEVA indico diferencias significativas en todo el periodo del cultivo. La curva de acumulación de calcio (Figura 4) muestra un comportamiento ascendente desde los 20 hasta los 100 y 120 dds respectivamente, debido que este elemento es esencial para el crecimiento de las raíces y como un constituyente del tejido celular de las membranas (8). La variedad que más acumula Ca es Imbaya con 61,92 kg ha-1, seguida de la variedad Ecu-324 con 52,94 kg ha-1 y la variedad Chasqui la que menos acumula calcio con 49,58 kg ha-1.

La acumulación de calcio fue mayor en la panoja con 32,01 kg ha-1. De acuerdo con FAO (4) señala que la quinua es un alimento que contiene 148,7 mg de Ca por cada $100 \mathrm{~g}$ de peso en seco. En menor cantidad en las hojas con $17,84 \mathrm{~kg}$ ha-1 y en el tallo con $12,07 \mathrm{~kg}$ ha-1. En las hojas a partir de los 80 dds se observa un pequeño descenso en la acumulación de Ca. 


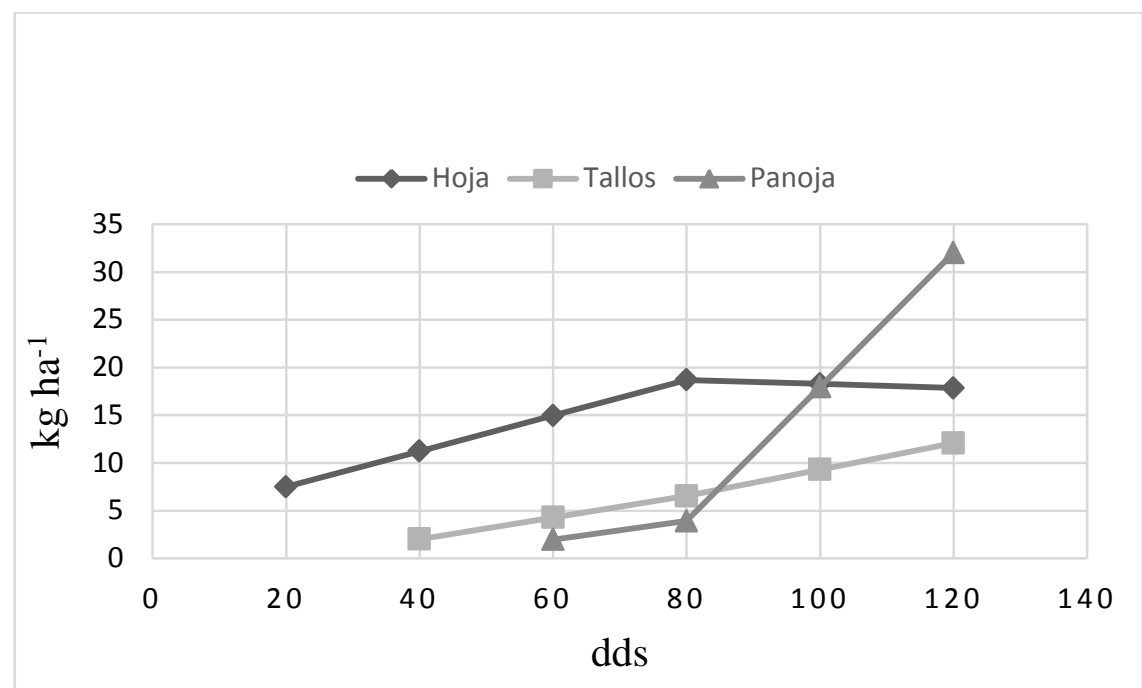

Figura 4. Acumulación de Ca en planta de quinua (Chenopodium quinoa Willd), variedad Imbaya.

\section{Acumulación de magnesio (Mg)}

El análisis de varianza determino que existieron diferencias significativas durante todos los muestreos. La acumulación de magnesio (Figura 5) se presenta en dos periodos; de 20 a 40 dds la acumulación es baja, mientras que a partir de los 40 dds hasta los 100 y 120 dds respectivamente la acumulación se incrementa notablemente conforme se desarrolla la planta. Esto se debe según CARE (8), a que el Mg es el constituyente central de la clorofila; por ello, del 15 al $20 \%$ del magnesio contenido en la planta se encuentra en las partes verdes. El $\mathrm{Mg}$ se incluye también en las reacciones enzimáticas relacionadas a la transferencia de energía en la planta. (17).

Como se observa en la Tabla 5, la variedad que más acumula magnesio es la Imbaya con
58,17 kg ha-1, seguida de la variedad Ecu-324 con $51,57 \mathrm{~kg}$ ha-1 y la variedad Chasqui con $50,64 \mathrm{~kg}$ ha-1. Como se observa en la tabla 6, la fertilización con N, P, K en dosis de 150-80-40 permitió una mayor acumulación de $\mathrm{Mg}$ de $64,77 \mathrm{Kg}$, demostrándose con esto que existe un efecto de sinergismo con el N (17). Mientras que la testigo solamente acumulo $36,8 \mathrm{~kg}$.

La curva de absorción de Mg fue ascendente (4) en todos los muestreos y en todos los órganos. La panoja acumuló $37,76 \mathrm{~kg}$ ha-1, esto se debe a que este órgano alberga a los granos los cuales según la FAO (4), señala que la quinua aporta una importante cantidad de magnesio, con un contenido de 249,6 mg por cada $100 \mathrm{~g}$ de peso en seco; seguido de las hojas con 17,98 kg ha-1 y en los tallos con 8,43 kg ha-1. 


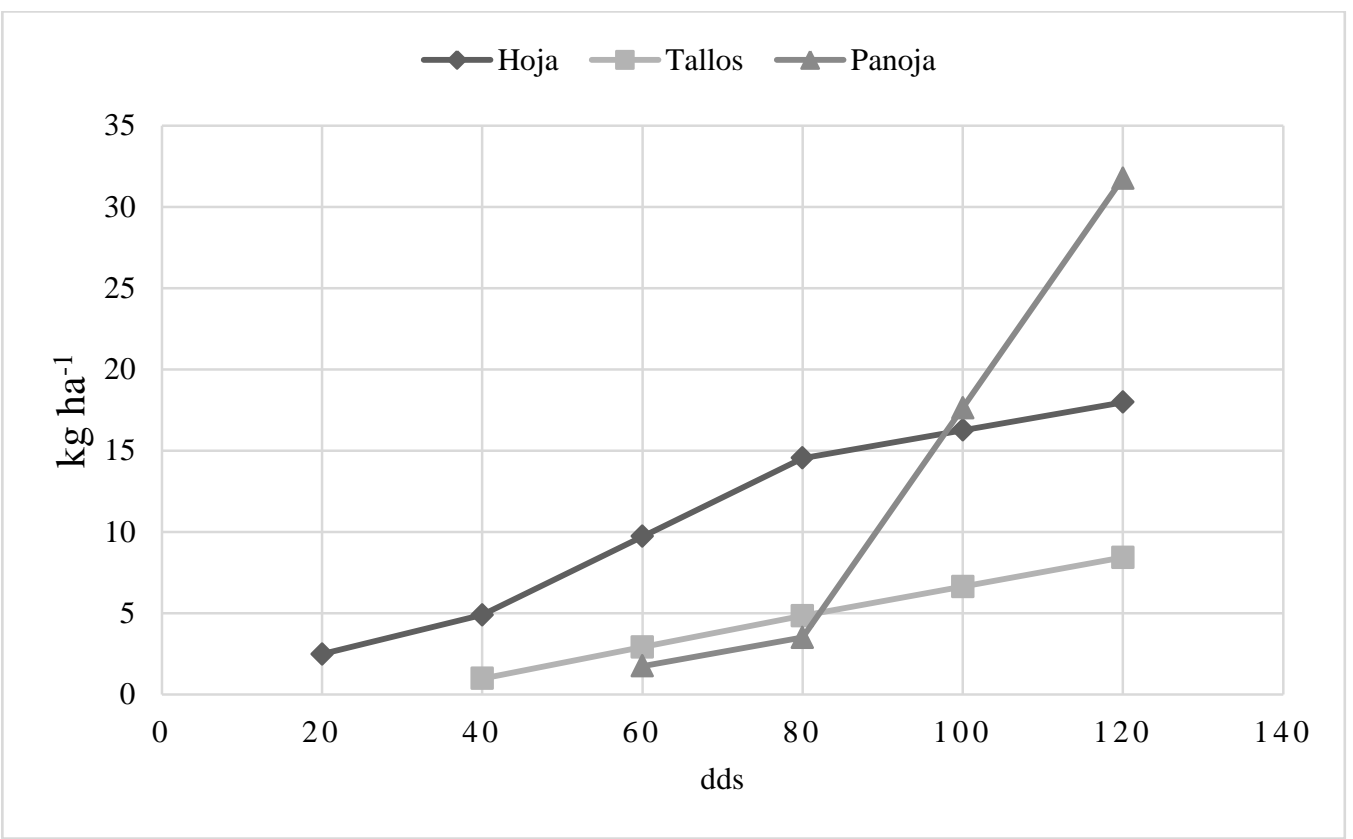

Figura 5. Acumulación de Mg en planta de quinua (Chenopodium quinoa Willd), variedad Imbaya

Tabla 5. Acumulación de nutrientes en $\mathrm{kg} \mathrm{ha}^{-1}$ de tres variedades de quinua (Chenopodium quinoa Willd).

\begin{tabular}{lccccc}
\hline Variedad & $\mathbf{N}$ & $\mathbf{P}$ & $\mathbf{K}$ & $\mathbf{C a}$ & $\mathbf{M g}$ \\
\hline Chasqui & 201,73 & 37,55 & 206,01 & 61,92 & 50,64 \\
Imbaya & 141,14 & 37,8 & 220,78 & 52,94 & 58,17 \\
Ecu-324 & 87,6 & 41,29 & 341,5 & 49,58 & 51,57 \\
\hline
\end{tabular}

Promedios con la misma letra dentro de cada factor son iguales estadísticamente, Tukey $(\mathrm{P}=0.05)$

Tabla 6. Acumulación de nutrientes en $\mathrm{kg} \mathrm{ha}^{-1}$ con tres niveles de fertilización aplicadas al cultivo de quinua (Chenopodium quinoa Willd).

\begin{tabular}{lccccc}
\hline \multicolumn{1}{c}{ Niveles de fertilización } & $\mathbf{N}$ & $\mathbf{P}$ & $\mathbf{K}$ & $\mathbf{C a}$ & $\mathbf{M g}$ \\
\hline $\mathbf{0 - 0 - 0}$ & 80,66 & 15,82 & 124,37 & 38,5 & 36,8 \\
$\mathbf{7 5 - 4 0 - 2 0}$ & 144,25 & 36,28 & 166,57 & 58,63 & 58,11 \\
$\mathbf{1 5 0 - 8 0 - 4 0}$ & 204,33 & 52,38 & 337,39 & 67,2 & 64,77 \\
\hline
\end{tabular}

Promedios con la misma letra dentro de cada factor, son iguales estadísticamente, Tukey ( $\mathrm{P}=0.05)$.

En Índice de Cosecha para la materia seca se obtuvo dividiendo la materia seca del grano para la materia seca de la planta total, obteniéndose valores de: 0,57 para la variedad Chasqui, 0,52 para la variedad
Imbaya y 0,53 para la variedad Ecu-324, sin presentarse diferencias significativas. En la Tabla 7 se demuestran los valores del Índice de Cosecha para la variedad Imbaya con N, P, $\mathrm{K}$, Ca y $\mathrm{Mg}$, en donde se observa que los 
valores de 0,55 para $\mathrm{K}$ y 0,52 para Ca son los menores. Zamudio-Gonzalez, et al (18) encontraron valores de Índices de Cosecha en maíz, con $\mathrm{N}=0,45 ; \mathrm{P}=0,75 ; \mathrm{K}=0,21 ; \mathrm{Mg}=0,45$ y $\mathrm{S}=0,43$, observándose que para quinua tenemos valores mayores excepto en $P$.

Tabla 7. Índices de cosecha (IC) con N, P, K, Ca, Mg en la variedad Imbaya.

\begin{tabular}{cccccc}
\hline V.Imbaya & $\mathbf{N}$ & $\mathbf{P}$ & $\mathbf{K}$ & $\mathbf{C a}$ & $\mathbf{M g}$ \\
\hline Grano & 88.91 & 24,20 & 121,43 & 37.72 & 37.82 \\
Planta & 141,14 & 37,80 & 220,78 & 61,02 & 58,17 \\
I.C. & 0,63 & 0,64 & 0,55 & 0,52 & 0,65 \\
\hline
\end{tabular}

\section{CONCLUSIONES}

Bajo las condiciones del ensayo se determinó que existen diferencias en la dinámica de absorción de nutrientes en las tres variedades de quinua en estudio.

El rendimiento de las tres variedades de quinua estuvo en función de la fertilización aplicada al suelo, presentando un mejor rendimiento la fertilización con 150-80-40 kg ha-1 de N, P, K.

Los nutrientes más absorbidos por la planta de quinua fueron $\mathrm{K}$ y $\mathrm{N}$ seguido de $\mathrm{Ca}$, Mg y P en este orden.

En la planta, el movimiento de nutrientes hacia el grano, desde las hojas y tallo se da con mayor intensidad a partir de los 80 días después de la siembra.

Se recomienda hacer las aplicaciones de fertilizantes basados en la absorción de nutrientes y las expectativas de rendimiento de cada una de las variedades, pues a mayor rendimiento mayor demanda de nutrientes.

Los Índices de Cosecha para la materia seca fueron: 0,57 para la variedad Chasqui, 0,52 para la variedad Imbaya y 0,53 para la variedad Ecu-324, y los índices de cosecha con macrolementos fueron: con $\mathrm{N}=0,63$, con $\mathrm{P}=0,64$, con $\mathrm{K}=0,55$, con $\mathrm{Ca}=0,52$ y con $\mathrm{Mg}=0,65$

\section{REFERENCIAS}

1. Peralta E. La quinua en Ecuador. 2009. [en línea]. Disponible en: http://www.iniap.gob.ec/nsite/images/docu mentos/ESTAD0\%20DEL\%20ARTE\%20QUI NUA\%202.pdf. [Consulta: febrero 10, 2019].

2. Reyes Montaño, EA, Ávila Torres, DP, Guevara Pulido JO. Componente nutricional de diferentes variedades de quinua de la región Andina. AVANCES Investigación en Ingeniería. 2006; 5: 86-97.

3. Repo-Carrasco-Valencia, R. and Serna L.A. Quinoa (Chenopodium quinua, Willd.) as a source of dietary fiber and other functional components." Ciencia e Tecnología de Alimentos. 2011. 19 (1): 225-230.

4. FAO. Año internacional de la quinua. Chile. $2013 . \quad$ Disponible en http://www.fao.org/quinoa-2013/what-isquinoa/nutritional-value/es/. Consulta: 24 de febrero de 2019.

5. FAO. La quinua: cultivo milenario para contribuir a la seguridad alimentaria mundial. 2011. Italia. Disponible en http://www.fao.org/fileadmin/templates/aiq 2013/res/es/cultivo_quinua_es.pdf.

[Consulta: febrero 20, 2017].

6. Peralta EN, Mazón A, Murillo M, Rivera D, Rodríguez L, Lomas CM. Manual Agrícola de Granos Andinos: Chocho, Quinua, Amaranto y Ataco. Cultivos, variedades y costos de producción. 2012 Tercera edición. 
Publicación Miscelánea $\mathrm{N}^{\circ}$ 69. Programa Nacional de Leguminosas y Granos Andinos. Estación Experimental Santa Catalina. INIAP. Quito, Ecuador. 68p.

7. FAO y Universidad Nacional Agraria La Molina. Guía del cultivo de quinua. 2016. Lima-Perú. Disponible

http://www.fao.org/3/a-i5374s.pdf.

Consulta: 03 de marzo de 2019.

8. CARE. Manual de fertilización y nutrición de la quinua. Primera edición. Lima Perú. 2012 Disponible

http://www.care.org.pe/wpcontent/uploads/2015/06/Manual-deFertilizacion-de-La-Quinua.pdf. (Consulta: febrero 12, 2019).

9. SOLID, OPD. Tecnología productiva de la quinua. $2010 . \quad$ Disponible en: file://F:/UTA\%202017/Articulos\%20científ icos/Art.\%20Cient/TECNOLOGÍA\%20PRODU CTIVA\%20DE\%20LA\%20QUINUA.pdf. Consulta: febrero 17, 2019.

10. Calvache-Ulloa AM, Libardi PL, Reichardt K. Curvas de absorción del Fertilizante Nitrogenado por dos híbridos de maíz. Fundación Cargil, Campinas, Brasil. 1981; 120.

11. Bertsch, F. Utilidad de los estudios de absorción de nutrientes en el afinamiento de las recomendaciones de fertilización. 2008. P 1. En VIII Congreso Ecuatoriano de la Ciencia del Suelo. Octubre. Quito, Ecuador.
12. INTAGRI. Las curvas de absorción de nutrientes, Guanajuato, México. 2016. Disponible en https://www.intagri.com/articulos/nutricion -vegetal/las-curvas-de-absorcion-de-

nutrientes\#sthash.v0NK1vGS.dpbs (Consulta 23 marzo 2019).

13. Ciampitti I, García FO. Requerimientos nutricionales: Absorción y extracción de macronutrientes y nutrientes secundarios. Hortalizas, frutales $\mathrm{y}$ forrajes. Archivos agronómicos, 2008; 12: 1-4.

14. Calvache -Ulloa AM. Los Suelos del Ecuador. VI Congreso Latinoamericano de Agronomía. 2008. Guayaquil-Ecuador. 2015

15. Solomon EL, Berg, Martin D. Biología, Novena edición. Cengage Learning. México. DF. 2016; 1321.

16. Villavicencio A, Vásquez C. Wilson. Eds. Guía Técnica de cultivos. 2008. Quito, EC, INIAP. 444 p. (Manual № 73).

17. Calvache- Ulloa, AM. Nutrimentos de Plantas. 2013. Universidad Central del Ecuador 420p.

18. Zamudio-Gonzales $B$, Tadeo-Robledo $M$, Espinosa-Calderón A, Martinez-Rodriguez N, Turrent-Fernandez A. Índice de Cosecha com macronutrimentos em grano de maiz. Revista Mexicana de Ciencias Agricolas. 2016. 7:10771089. 